NISSUNA UMANA INVESTIGAZIONE SI PUO DIMANDARE VERA SCIENZIA S'ESSA NON PASSA PER LE MATEMATICHE DIMOSTRAZIONI LEONARDO DA VINCI

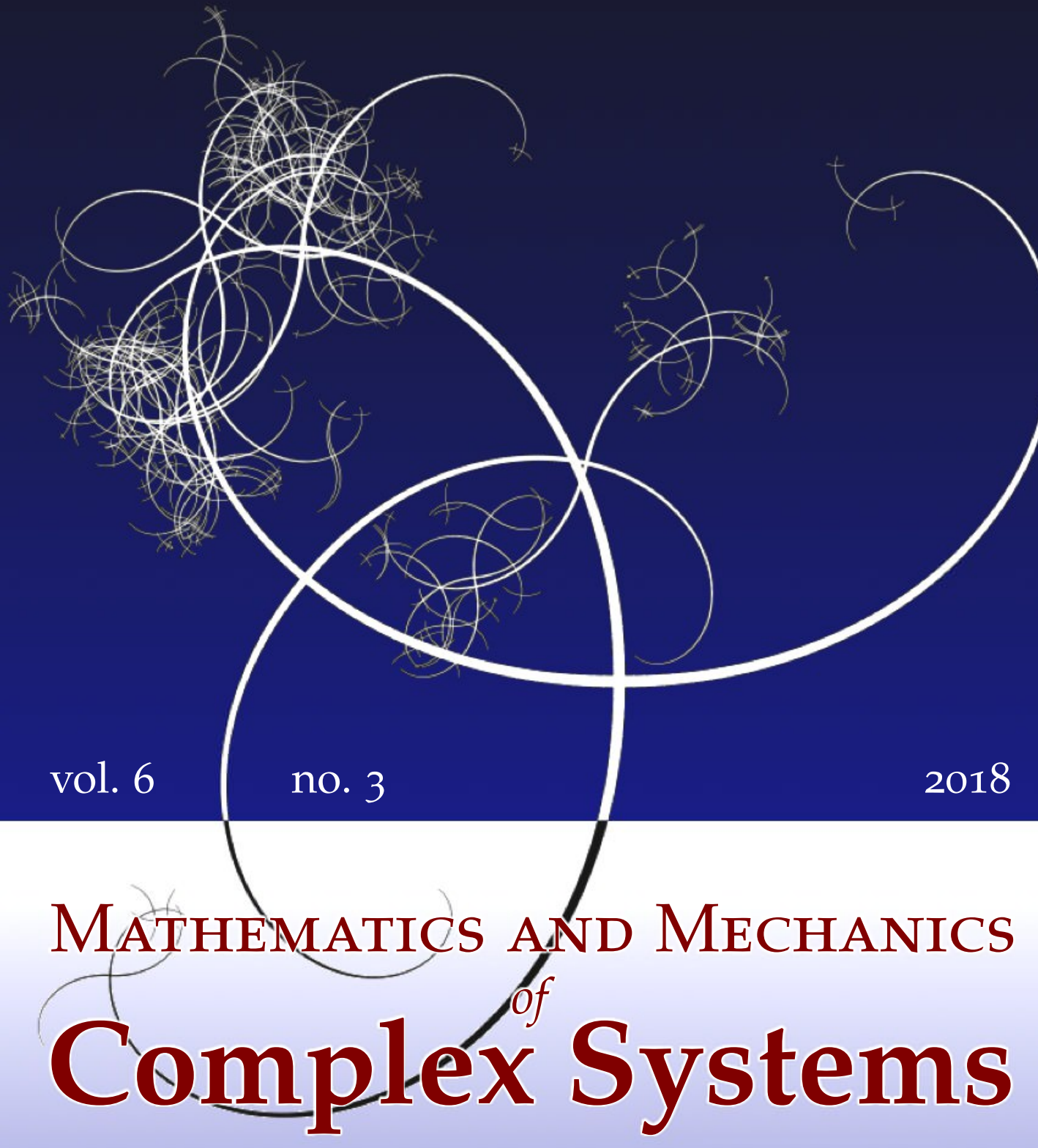

ELENA LOPEZ, ADRIEN SCHEUER,

Emmanuelle Abisset-Chavanne AND Francisco Chinesta

ON THE EFFECT OF PHASE TRANSITION ON THE MANIFOLD DIMENSIONALITY: APPLICATION TO THE ISING MODEL 


\title{
ON THE EFFECT OF PHASE TRANSITION ON THE MANIFOLD DIMENSIONALITY: APPLICATION TO THE ISING MODEL
}

\author{
ELENA LOPEZ, ADRIEN SCHEUER, \\ Emmanuelle Abisset-Chavanne ANd Francisco Chinesta
}

\begin{abstract}
Fields can be represented in a discrete manner from their values at some locations, the nodes when considering finite element descriptions. Thus, each discrete scalar solution can be considered as a point in $\mathbb{R}^{N}$ ( $N$ being the number of nodes used for approximating the scalar field). Most manifold learning techniques (linear and nonlinear) are based on the fact that those solutions define a slow manifold of dimension $n \ll N$ embedded in the space $\mathbb{R}^{N}$. This paper explores such a behavior in systems exhibiting phase transitions in order to analyze the evolution of the local dimensionality $n$ when the system moves from one side of the critical behavior to the other. For that purpose we consider the Ising model.
\end{abstract}

\section{Introduction}

Physical models usually involve unknown continuous scalar or vector fields. If we consider without any loss of generality a model involving a scalar field approximated on a mesh or grid involving $N$ nodes, the discrete solution can be represented as a point belonging to $\mathbb{R}^{N}$. However, existing correlations lead to solutions that, instead of filling the whole space $\mathbb{R}^{N}$, define a slow $n$-dimensional manifold, with $n \ll N$, embedded into $\mathbb{R}^{N}$.

This fact is at the origin of manifold learning approaches that consist of extracting the uncorrelated (latent) dimensions describing the slow manifold representing the original states into a reduced form. Principal component analysis (PCA) was specially designed to find a linear subspace of lower dimensionality than the original space; however, it fails when the manifold becomes extremely nonlinear. Nonlinear dimensionality reduction techniques were proposed for circumventing this limitation. Among the many existing techniques (an abundant literature is available on the topic, and the interested reader can refer to [Lee and Verleysen 2007] and the references therein) kernel-based PCA (kPCA) [Wang 2014; Schölkopf et al.

\section{Communicated by Francesco dell'Isola.}

PACS2010: 05.10.Ln.

Keywords: Ising equation, phase transition, manifold learning. 
1998; 1999] and locally linear embedding (LLE) [Roweis and Saul 2000] will be considered in the present work. The latter can be considered as a particular case of the former, for a particular kernel choice [Zimmer et al. 2015].

These techniques that automatically extract the latent dimensions have been extensively and successfully applied in many domains; however, few works addressed the issue of phase transition and in particular the way in which that transition affects the manifold dimensionality and the possibility of characterizing the transition from a geometrical point of view. To address that issue, we consider in the present work a well established and widely studied equation exhibiting phase transition, the Ising model.

In the context of the microscopic theory of critical behavior [Koonin and Meredith 1990; Fisher 1965], the Ising model [Newell and Montroll 1953] addresses a ferromagnetic lattice in a quite simple statistical description, including phase transition. Ferromagnetic materials exhibit long-range spin ordering at the atomic level. When a magnetic field is applied to a ferromagnetic material, atomic spins align along the direction of the applied field. However, when the temperature becomes higher than the so-called critical temperature, a phase change occurs. The Ising model allows describing such materials [Myers 1997]. This model initially proposed by Lenz was solved in 1D by Ising [1925]. The 2D model without magnetic field was then developed by Onsager [1944], and it is this one that we are considering in the present work. Its solution that will be addressed by using the Monte Carlo method [Metropolis et al. 1953] concerns a binary property called spin, given each point of a 2D grid, and more specifically its time evolution with respect to the applied temperature. When reaching the so-called critical temperature, phase transition occurs. Even if complex variants of the Ising model were proposed [Bellettini et al. 2007; De Masi et al. 2009; 2008], in the present work we consider its simplest version.

Our feeling is that applying usual manifold learning techniques on the spin field itself, consisting of a binary variable defined at each grid (mesh) node, is neither the best nor the optimal representation because those techniques are based on the use of euclidean metrics and two similar microstructures can significantly differ when calculating the euclidean distance between both of them. For that reason, a first contribution of the present work is to propose a better microstructure representation much less sensitive to local distributions. In that sense, considering Fourier-based representations, where space dependencies are described from their frequency content, seems a much better alternative, in particular, the 2D fast Fourier transform (FFT) 2D-FFT that has been widely considered in image analysis to identify biological damage [Fung et al. 2010], extract ordered structures from microtomography [Jeulin and Moreaud 2008], analyze complex microstructures [Lebensohn et al. 2011; Zhu et al. 2018], etc. 
When applying the FFT to solutions coming from the Ising model, one expects low frequencies at low temperatures, where long-range correlation exists, whereas at high temperatures, where entropic effects dominate, higher frequency contents are expected. However, at the transition in between, richer structures with its a priori higher dimensionality are expected. Thus, we would like to locally define the manifold dimensionality, and instead of using global manifold learning techniques, local extractors seem more appropriate. In particular local principal component analysis (IPCA) [Kambhatla and Leen 1997] seems specially appealing for that purpose. It is important to note that IPCA encountered limited interest as a manifold learning technique because of the difficulty of defining a continuous mapping throughout the manifold. However, it has been widely employed in other domains where the main goal, as in the present case, was to estimate the local dimensionality [Fukunaga and Olsen 1971].

The paper is structured as follows. Section 2 revisits the main concepts related to the Ising model, as well as its solution using the Metropolis-based Monte Carlo algorithm. Section 3 addresses the application of LLE for constructing the manifold on which data will be classified using the procedure proposed in Section 4. Finally, Section 5 presents and discusses different numerical solutions, proving the potential of the proposed approach.

\section{The Ising model and its Monte Carlo solution}

The Ising model describes a ferromagnetic behavior and is able to capture phase transitions [Niss 2005]. In this model, the discrete variables called "spins" are defined on a lattice. Each lattice site has only one spin with value either -1 (spin down) or +1 (spin up), interacting only with its nearest neighbors. By considering the 2D Ising model introduced by Onsager [1944], the Hamiltonian $H$ reads

$$
H=-J \sum_{i} \sum_{\substack{j \\|i-j|=1}} S_{i} S_{j}
$$

$J$ being the dimensionless interaction strength. $S_{i}$ and $S_{j}$ are the spin states at lattice sites $i$ and $j$, respectively. As can be noticed, the sum of products reduces to the nearest-neighbor pair of spins. Every spin will interact with four other spins (up, down, left, and right). If spins are aligned and $J>0$, the energy of the system will be minimal.

The partition function $Z$ can be computed from the Hamiltonian

$$
Z=\sum_{i} e^{-H_{i}}
$$


where the sum applies over all the particles in the lattice. Then, we compute the probability of finding the system in a particular state $S$ from

$$
p(\boldsymbol{S})=\frac{e^{-H(S)}}{Z} .
$$

Some important outputs are the magnetization $\mathrm{Ma}$

$$
\mathrm{Ma}=\sum_{S} p(S) \sum_{i} S_{i},
$$

and the system energy $E$

$$
E=\sum_{S} p(\boldsymbol{S}) \beta H(\boldsymbol{S}),
$$

where $\beta=k_{B} T$, with the Boltzmann constant $k_{B}=1.380658 \times 10^{-23} \mathrm{~J} / \mathrm{K}$ and $T$ the temperature.

Usually the solution of the Ising model is performed by using a Monte Carlo method. In this paper, we consider a variant of the Monte Carlo method, the socalled Metropolis algorithm [Fricke 2006]. For a given temperature, at each time step, a trial spin configuration is generated. The algorithm computes the system energy associated with the trial state. If the change of energy is negative, it means that the system evolution has brought the system to a state of lower energy, so we allow the change and put the spins in their new state. On the other hand, if the change of energy is positive, we allow the change with a probability given by $p(\boldsymbol{S})$ computed from (3).

\section{Manifold construction}

It is well known that microstructures do not allow simple reduced descriptions [Lopez et al. 2018]. In fact the main concern is how to quantify similarities or resemblances, and how to take profit of them.

In this paper we consider and analyze a route based on the use of the locally linear embedding (LLE) technique [Roweis and Saul 2000], a member of the large family of the so-called manifold learning techniques.

The remainder of this section describes the procedure directly on the problem we are interested in. The procedure consists of two steps: the analysis of the Ising samples in order to obtain a discriminative description of the microstructures (in this case as previously said, we do not work directly on the Ising microstructures but on their fast Fourier transform), followed by a dimensionality reduction able to discriminate the three temperature zones that characterize the Ising model behavior.

3.1. Applying the fast Fourier transform on the Ising microstructures. First we assume the existence of $M$ microstructures $\mathcal{M}_{m}, m=1, \ldots, M$, coming from the 
Ising model defined on the domain $\omega$. In what follows and without loss of generality, we consider 2D microstructures and the 2D fast Fourier transform (2D-FFT). Moreover, we assume the existence of two phases, the spins with value 1 and the others with value -1 , occupying the domains $\omega_{1}^{m}$ and $\omega_{-1}^{m}$, respectively, with $\omega_{1}^{m} \cup \omega_{-1}^{m}=\omega, m=1, \ldots, M$. A regular mesh is associated with each microstructure consisting of $N$ nodes $\left(N=N_{n}^{2}\right.$, with $N_{n}$ the number of nodes along the $x$ and $y$ directions). The coordinates of each node are $\boldsymbol{x}_{i}, i=1, \ldots, N\left(\boldsymbol{x}_{i}^{T}=\left(x_{i}, y_{i}\right)\right)$.

For each microstructure $\mu_{m}$ we define the phase field $\chi\left(x ; M_{m}\right)$ as

$$
\chi\left(\boldsymbol{x} ; \mathcal{M}_{m}\right)=\left\{\begin{aligned}
1 & \text { if } \boldsymbol{x} \in \omega_{1}^{m}, \\
-1 & \text { if } \boldsymbol{x} \in \omega_{-1}^{m} .
\end{aligned}\right.
$$

As is well known, these microstructure descriptions do not allow simple reduced descriptions. Thus, the objective is to geometrically characterize them in order to obtain reduced descriptions. Concerning geometrical characterization and microstructural descriptions, there are several tools proposed for signal processing purposes. Concerning the type of microstructures we are dealing with, it seems more appropriate to work in the frequency domain instead of using their physical space description. In particular we propose to apply the 2D-FFT to characterize the microstructures in the frequency domain.

We now apply the 2D-FFT on each microstructure $\mu_{m}, m=1, \ldots, M$, defined by its phase field $\chi\left(\boldsymbol{x} ; \mathcal{M}_{m}\right)$. Thus, the 2D-FFT of the Ising microstructures can be represented in a discrete way from vectors $\hat{\chi}^{m}, \mu_{m}, m=1, \ldots, M$. Vectors $\hat{\chi}^{m}$ are defined in $\mathbb{R}^{N}$; i.e., the dimension coincides with the number of nodes considered in the discrete microscopic description.

3.2. Nonlinear dimensionality reduction. Each vector $\hat{\chi}^{m}$ defines a point in a space of dimension $N$, and then the data set of $M$ 2D-FFTs related to the $M$ Ising microstructures represents a set of $M$ points in $\mathbb{R}^{N}$. Hence, the question: do all these points belong to a particular low-dimensional manifold embedded in the high-dimensional space $\mathbb{R}^{N}$ ?

Imagine that, despite the impressive space dimension $N$, the $M$ points belong to a curve, a surface, or a hypersurface of dimension $n \ll N$. When $N=3$ a simple observation suffices to check if these points are located on a curve (1D manifold) or on a surface (2D manifold). However, when dealing with spaces of thousands of dimensions, simple visual observation is unsuitable.

Instead, appropriate techniques are needed to extract the underlying manifold (when it exists) when dealing with extremely multidimensional spaces. There is a variety of techniques to accomplish this task. The interested reader can refer to [Tenenbaum et al. 2000; Roweis and Saul 2000; Polito and Perona 2001; Wang 2014; Amsallem and Farhat 2008]. In this work we focus on the LLE (locally linear embedding) technology [Roweis and Saul 2000]. It proceeds as follows. 
- Each point $\hat{\chi}^{m}, m=1, \ldots, M$, is linearly reconstructed from its $K$-nearest neighbors. In principle $K$ should be greater than the expected dimension $n$ of the underlying manifold and the points should be close enough to ensure the validity of the linear approximation. In general, a large-enough number of neighbors $K$ and a dense-enough sampling $M$ ensure a satisfactory reconstruction as shown later. For each point $\hat{\chi}^{m}$ we can write the locally linear data reconstruction as

$$
\hat{\chi}^{m}=\sum_{i \in \mathscr{I}_{m}} W_{m i} \hat{\chi}^{i},
$$

where $W_{m i}$ are the unknown weights and $\mathscr{S}_{m}$ is the set of the $K$-nearest neighbors of $\hat{\chi}^{m}$.

Weights, grouped in vector $\boldsymbol{W}$, result from the minimization of functional

$$
\mathscr{F}(\boldsymbol{W})=\sum_{m=1}^{M}\left\|\hat{\chi}^{m}-\sum_{i=1}^{M} W_{m i} \hat{\chi}^{i}\right\|^{2},
$$

where here $W_{m i}$ is zero if $\hat{\chi}^{i}$ does not belong to the set of $K$-nearest neighbors of $\hat{\chi}^{m}$.

The minimization of $\mathscr{F}(\boldsymbol{W})$ allows us to determine all the weights involved in all the locally linear data reconstruction.

- We suppose now that each linear patch around $\hat{\chi}^{m}$, for all $m$, is mapped into a lower-dimensional embedding space of dimension $n, n \ll N$. Because of the linear mapping of each patch, weights remain unchanged. The problem now becomes the determination of the coordinates of each point $\hat{\chi}^{m}$ when it is mapped into the low-dimensional space, $\xi^{m} \in \mathbb{R}^{n}$.

For this purpose a new functional $\mathscr{G}$ is introduced that depends on the searched coordinates $\xi^{1}, \ldots, \xi^{M}$ :

$$
\mathscr{G}\left(\xi^{1}, \ldots, \boldsymbol{\xi}^{M}\right)=\sum_{m=1}^{M}\left\|\boldsymbol{\xi}^{m}-\sum_{i=1}^{M} W_{m i} \xi^{i}\right\|^{2},
$$

where now the weights are known and the reduced coordinates $\xi^{m}$ are unknown.

The minimization of functional $G$ results in an $M \times M$ eigenvalue problem whose $n$-bottom nonzero eigenvalues define the set of orthogonal coordinates in which the manifold is mapped.

\section{Discriminating criteria}

Once the manifold composed by the reduced coordinates $\xi^{m}$ is known, the goal is to cluster in some way the three temperature zones to be able to classify them 
in low, transition, and high temperature. For this purpose, we propose a criterion based on a variant of the principal component analysis (PCA), the so-called local principal component analysis (IPCA) that allows us to compute the manifold local dimension, and then infer the dimension at low, high, and critical temperatures.

Principal components analysis (PCA) attempts to find a linear subspace of lower dimensionality than the original space. If data exhibit more complex structures which cannot be well represented in a linear subspace, standard PCA fails to accomplish the reduction. However, such reduction can be successfully performed by using nonlinear dimensionality reduction techniques, like the IPCA here considered. In what follows we revisit first the standard PCA before focusing on its local counterpart.

Let us consider $n$ observed variables defining the vector (snapshot) $\xi \in \mathbb{R}^{n}$. We assume that these variables are therefore not totally uncorrelated and, notably, that there exists a linear transformation $\boldsymbol{L}$ defining the vector $\xi^{\text {red }} \in \mathbb{R}^{n^{\text {red }}}, n^{\text {red }} \leq n$, that represents the so-called latent reduced variables, according to

$$
\xi=L \xi^{\text {red }}
$$

We assume the existence of $M$ different snapshots $\xi_{1}, \ldots, \xi_{M}$ that can be stored in the columns of the $n \times M$ matrix $\mathbb{X}$. The associated $n^{\text {red }} \times M$ reduced matrix $\mathbb{Y}$ contains the associated reduced vectors $\xi_{i}^{\text {red }}, i=1, \ldots, M$.

PCA proceeds by enforcing the fact that latent variables must be as much as possible uncorrelated, and allows us to extract both the dimension $n^{\text {red }}$ and the mapping $\boldsymbol{L}$. For that purpose the covariance matrix $\boldsymbol{C}_{x x}$,

$$
C_{x x}=E\left\{(\mathbb{X}-E\{\mathbb{X}\})(\mathbb{X}-E\{\mathbb{X}\})^{T}\right\}
$$

is factorized as

$$
\boldsymbol{C}_{x x}=\boldsymbol{V} \boldsymbol{\Lambda} \boldsymbol{V}^{T},
$$

equivalent to applying the singular value decomposition (SVD) to $\mathbb{X}$. In (12), $\boldsymbol{V}$ contains the orthonormal eigenvectors and $\boldsymbol{\Lambda}$ is the diagonal matrix containing the eigenvalues (nonnegative real numbers), assumed to be in descending order. Thus, the $n^{\text {red }}$ columns of $\boldsymbol{L}$ are the $n^{\text {red }}$ first columns of $\boldsymbol{V}$ [Lee and Verleysen 2007].

From this summary of the PCA rationale, we can now briefly explain its local counterpart. For that purpose, we consider each snapshot $\xi_{i}$, for $i=1, \ldots, M$, and for each of them, its $K$-nearest neighbors. From those and by proceeding as just indicated, we can compute the local transformation matrix $\boldsymbol{L}_{i}, i=1, \ldots, M$, as well as the local reduced dimensionality $n_{i}^{\text {red }}$. 


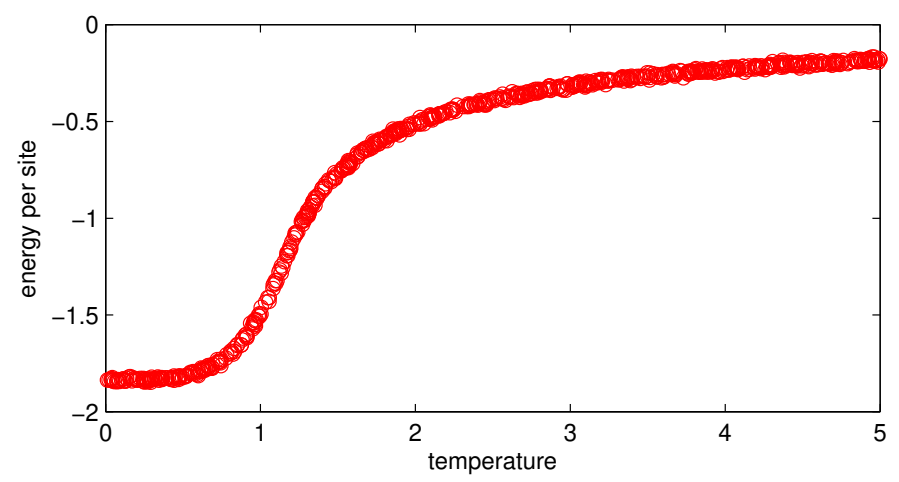

Figure 1. Energy versus temperature.

\section{Numerical results}

Ising microstructures have been generated using the Metropolis-based Monte Carlo method proposed in [Fricke 2006]. We have run $M=741$ simulations to obtain our microstructure data set (snapshots), $M_{m}, m=1, \ldots, M$. The parameters considered in those simulations are a $200 \times 200$ grid $(N=40000$ nodes $)$ with the dimensionless temperature $T$ randomly chosen between 0 and 5. Figure 1 shows the energy per site $E$ of the final configuration of each simulation. The magnetization per site in turn is shown in Figure 2.

It is clear that a phase transition occurs in between $T=1$ and $T=1.5$. At lower temperatures, $T<1$, the system tends to any of the two ground states $\mathrm{Ma}=-1$ or $\mathrm{Ma}=+1$. At higher temperatures, $T>1.5$, the spins tend to align randomly, leading to an almost vanishing magnetization $(\mathrm{Ma} \approx 0)$.

To illustrate the Ising microstructures configuration, Figure 3 depicts microstructures associated with low, transition, and high temperature. In the one associated with low temperature, approximately half of the spins are up and the other half

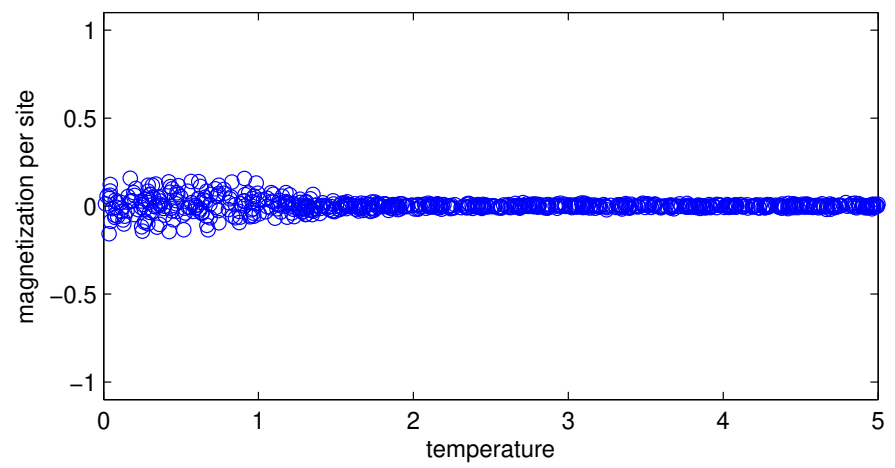

Figure 2. Magnetization versus temperature. 

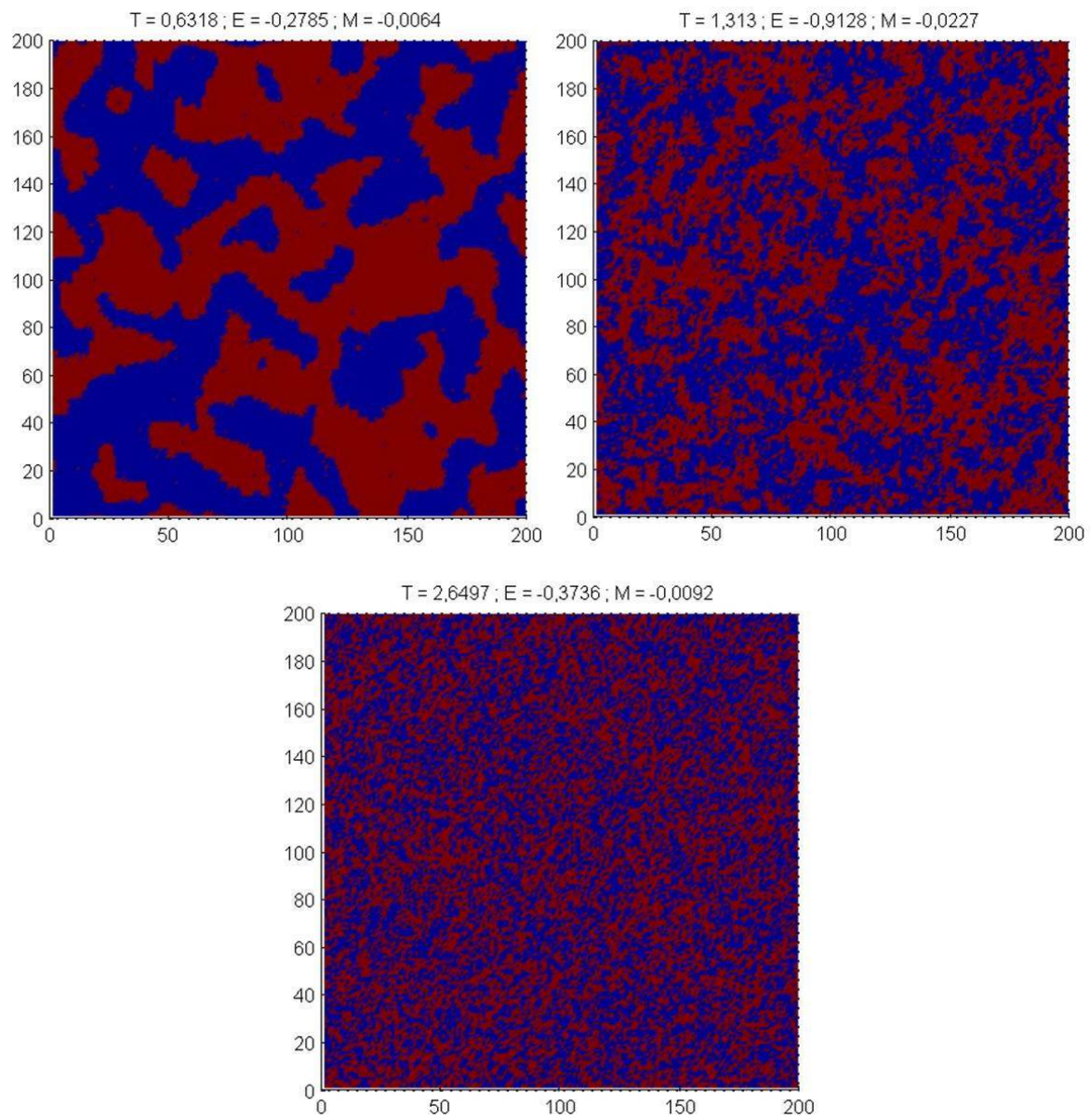

Figure 3. Low, transition, and high temperature microstructures (clockwise from top left).

down, with aligned spins forming a sort of clusters called "metastable states", with $\mathrm{Ma} \approx 0$. Around the transition temperature, the number of down spins has increased (up spins consequently decreasing) compared to the previous scenario. This means that the system has enough energy and the Metropolis algorithm easily accepts antiparallel spins. Finally, in the high-temperature state, almost the same number of down and up spins are found with a small characteristic length describing the phases distribution in the microstructures due to the high energy communicated to the system.

The $M$ samples were described by their phase field vectors $\chi^{m}, m=1, \ldots, M$, each defined in $\mathbb{R}^{N}$. But as explained in Section 3.1, microstructures do not allow 
reduced representations and that reason motivated the consideration of 2D-FFT on the $M$ Ising microstructures in order to obtain the data reduced representation.

Figure 4 illustrates the 2D-FFT representation of three microstructures associated with low, transition, and high temperature, respectively, considered in Figure 3. In the low-temperature microstructure, we can appreciate the presence of very few significant frequencies, almost located at the center of the domain (low frequencies), and the rest of the domain is practically flat.
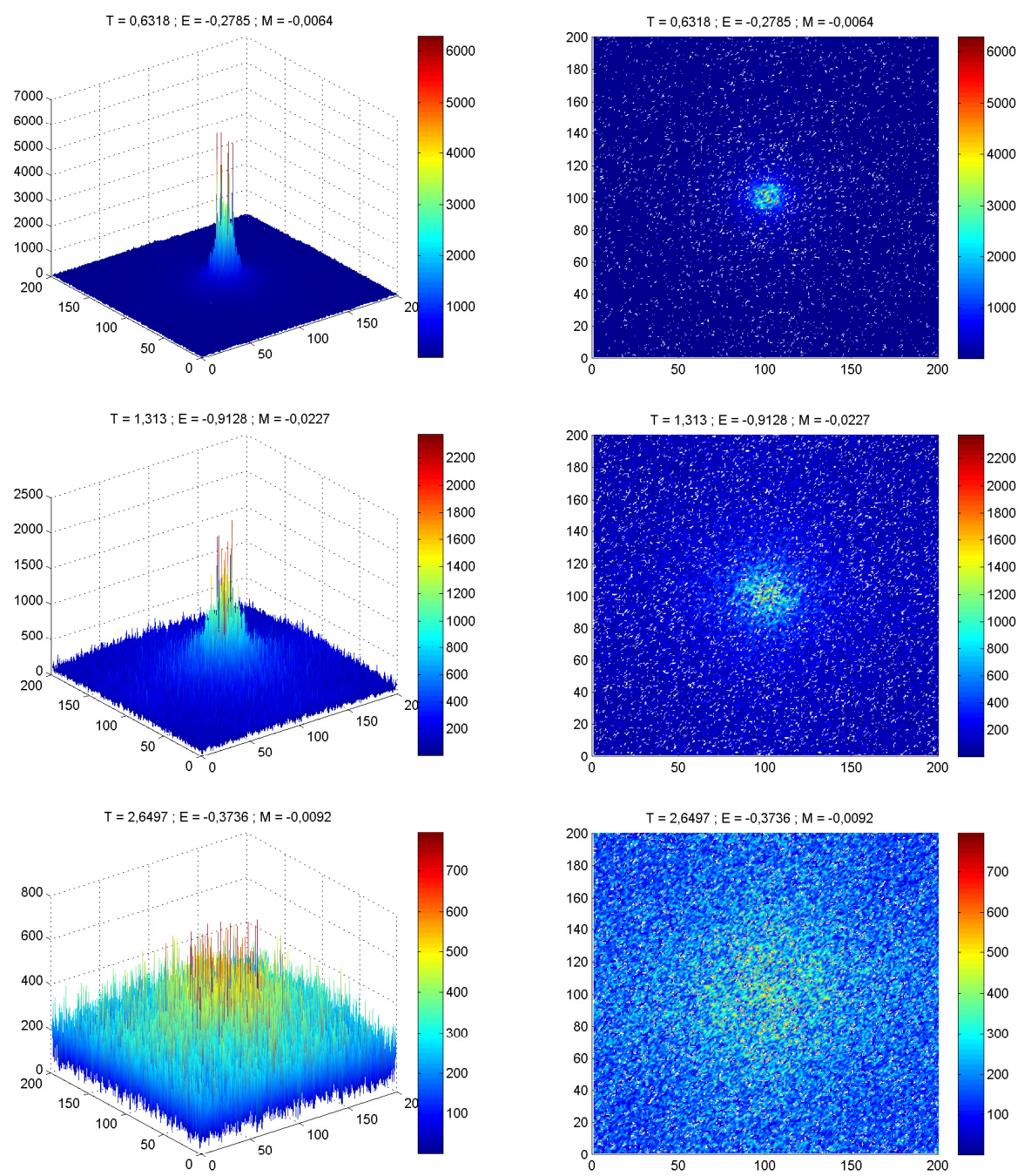

Figure 4. $3 \mathrm{D}$ (left) and 2D (right) representation of the 2D-FFT for low (top), transition (middle), and high (bottom) temperature microstructures. 
LLE manifold of the Fourier Transforms

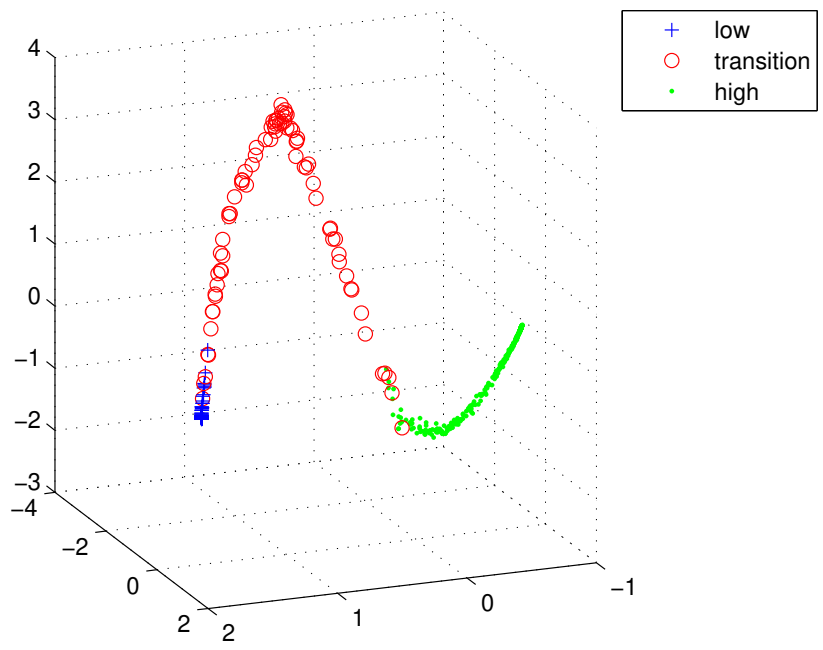

Figure 5. LLE manifold of the 2D-FFT on the Ising microstructures. Axes represent a 3D space in which the almost 1D manifold is embedded.

This result is intuitive since the spins of the microstructures are more clustered in the low-temperature samples. On the contrary, in the case of 2D-FFT applied to the high-temperature microstructures, we observe the opposite result. Here, spins are totally randomly distributed and consequently all frequencies are present. In the case of microstructures around the transition temperature, its 2D-FFT becomes a mix of the aforementioned cases, with dominant frequencies located at the center of the domain but now the rest of the domain is no longer flat, since it is populated by the contribution of nonnegligible higher frequencies.

We denote by $\hat{\chi}^{m}, m=1, \ldots, M$, the 2D-FFT of the Ising microstructures. By applying the locally linear embedding technique on $\hat{\chi}^{m}$, as explained in Section 3.2, the weights involved in the linear data reconstruction are calculated as well as the reduced data. The performed analysis allows us to consider a reduced dimension $n=3$ that moreover facilitates the solution's graphical representation. Figure 5 depicts the resulting points $\xi^{m} \in \mathbb{R}^{3}, m=1, \ldots, M$, from which one can realize that the manifold is almost $1 \mathrm{D}$.

Now in order to properly check the local dimensionality, local principal component analysis is applied in locations belonging to low, high, and transition temperatures, whose associated regions can be clearly identified in Figure 5. After applying the IPCA in these three regions (the solution represented in the manifold of Figure 5), the highest eigenvalues are extracted determining the local manifold dimensionality. As shown in Figure 6, the local dimensionality of low and high 

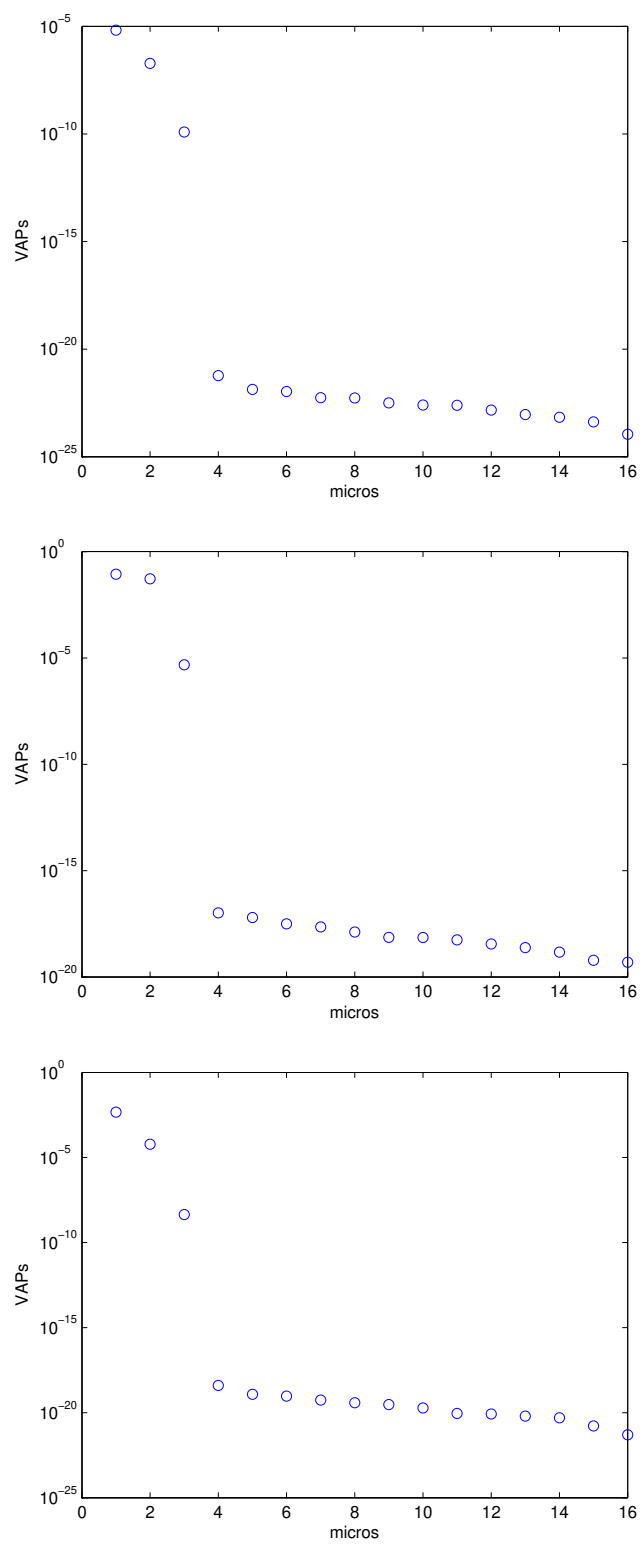

Figure 6. Local dimensionality of the low, transition, and high temperature zones (top to bottom).

temperatures is one because there is a difference of almost two orders of magnitude between the values of the first and the second eigenvalues, which means that the first is much more important than the second.

This result was expected, since looking at the manifold of Figure 5, it could be clearly seen that the points corresponding to these two zones defined a rather 
1D manifold. In the transition region the first two eigenvalues have practically the same value and differ from the third by four orders of magnitude, which clearly means that the local dimensionality of this region is almost two. This result was also expected, since the points in this zone mix the 1D behaviors of low and high temperatures and being not collinear increases the dimensionality. Local dimension is not able to distinguish between the two phases of the system. Descriptions able to differentiate them constitute a work in progress.

\section{Conclusions}

This paper proposes a methodology to interpret phase transition from a geometrical point of view, from the local dimensionality of the manifold defined from different microscopic fields. For that purpose the Ising model is simulated by varying the temperature from one side to other of the critical temperature associated with the phase transition.

Using the Metropolis algorithm, a group of microstructures related to the solution of the Ising model has been created, encompassing samples corresponding to low, high, and transition temperatures. Unable to deal directly with the microstructures' characteristic function, the 2D-FFT has been applied to those because the frequency content is expected to exacerbate the difference between microstructures at low, high, and transition temperatures. The manifold was then created on the 2D-FFT representations of phases distribution, and then a local PCA was applied locally in the different regions to extract the local dimensionality.

The performed analysis reveals that the dimensionality slightly increases in the transition region where complex microstructures mixing low and high temperature patterns coexist. Below and above that transition temperature the solution seems to be explained by only one latent variable that in the present case can be associated with the temperature. In the transition region solutions seem a bit richer and cannot be explained by a single latent variable.

\section{References}

[Amsallem and Farhat 2008] D. Amsallem and C. Farhat, "Interpolation method for adapting reducedorder models and application to aeroelasticity", AIAA J. 46:7 (2008), 1803-1813.

[Bellettini et al. 2007] G. Bellettini, A. De Masi, N. Dirr, and E. Presutti, "Tunneling in two dimensions", Comm. Math. Phys. 269:3 (2007), 715-763.

[De Masi et al. 2008] A. De Masi, I. Merola, E. Presutti, and Y. Vignaud, "Potts models in the continuum: uniqueness and exponential decay in the restricted ensembles", J. Stat. Phys. 133:2 (2008), 281-345.

[De Masi et al. 2009] A. De Masi, I. Merola, E. Presutti, and Y. Vignaud, "Coexistence of ordered and disordered phases in Potts models in the continuum", J. Stat. Phys. 134:2 (2009), 243-306.

[Fisher 1965] M. E. Fisher, "The nature of critical points", pp. 1-159 in Lectures in theoretical physics, vol. VIIC, edited by W. E. Brittin, University of Colorado, 1965. 
[Fricke 2006] T. Fricke, "Monte Carlo investigation of the Ising model", preprint, 2006, Available at https://www.physics.ohio-state.edu/ braaten/statphys/Ising_MatLab.pdf.

[Fukunaga and Olsen 1971] K. Fukunaga and D. R. Olsen, "An algorithm for finding intrinsic dimensionality of data”, IEEE T. Comput. C-20:2 (1971), 176-183.

[Fung et al. 2010] D. T. Fung, J. B. Sereysky, J. Basta-Pljakic, D. M. Laudier, R. Huq, K. J. Jepsen, M. B. Schaffler, and E. L. Flatow, "Second harmonic generation imaging and Fourier transform spectral analysis reveal damage in fatigue-loaded tendons", Ann. Biomed. Eng. 38:5 (2010), 17411751.

[Ising 1925] E. Ising, "Beitrag zur Theorie des Ferromagnetismus", Z. Phys. 31 (1925), 253-258.

[Jeulin and Moreaud 2008] D. Jeulin and M. Moreaud, "Segmentation of 2D and 3D textures from estimates of the local orientation", Image Anal. Stereol. 27:3 (2008), 183-192.

[Kambhatla and Leen 1997] N. Kambhatla and T. K. Leen, "Dimension reduction by local principal component analysis", Neural Comput. 9:7 (1997), 1493-1516.

[Koonin and Meredith 1990] S. E. Koonin and D. Meredith, Computational physics: FORTRAN version, Addison-Wesley, 1990.

[Lebensohn et al. 2011] R. A. Lebensohn, A. D. Rollett, and P. Suquet, "Fast Fourier transformbased modeling for the determination of micromechanical fields in polycrystals", J. Mater. 63:3 (2011), 13-18.

[Lee and Verleysen 2007] J. A. Lee and M. Verleysen, Nonlinear dimensionality reduction, Springer, 2007.

[Lopez et al. 2018] E. Lopez, D. Gonzalez, J. V. Aguado, E. Abisset-Chavanne, E. Cueto, C. Binetruy, and F. Chinesta, "A manifold learning approach for integrated computational materials engineering", Arch. Comput. Methods Eng. 25:1 (2018), 59-68.

[Metropolis et al. 1953] N. Metropolis, A. W. Rosenbluth, M. N. Rosenbluth, and A. H. Teller, "Equation of state calculations by fast computing machines", J. Chem. Phys. 21:6 (1953), 10871092.

[Myers 1997] H. P. Myers, Introductory solid state physics, 2nd ed., Taylor \& Francis, 1997.

[Newell and Montroll 1953] G. F. Newell and E. W. Montroll, "On the theory of the Ising model of ferromagnetism”, Rev. Modern Physics 25 (1953), 353-389.

[Niss 2005] M. Niss, "History of the Lenz-Ising model 1920-1950: from ferromagnetic to cooperative phenomena", Arch. Hist. Exact Sci. 59:3 (2005), 267-318.

[Onsager 1944] L. Onsager, "Crystal statistics, I: A two-dimensional model with an order-disorder transition”, Phys. Rev. (2) 65 (1944), 117-149.

[Polito and Perona 2001] M. Polito and P. Perona, "Grouping and dimensionality reduction by locally linear embedding", pp. 1255-1262 in Advances in Neural Information Processing Systems 14 (Vancouver, 2001), edited by T. G. Dietterich et al., MIT, 2001.

[Roweis and Saul 2000] S. T. Roweis and L. K. Saul, "Nonlinear dimensionality reduction by locally linear embedding”, Science 290:5500 (2000), 2323-2326.

[Schölkopf et al. 1998] B. Schölkopf, A. Smola, and K.-R. Müller, "Nonlinear component analysis as a kernel eigenvalue problem", Neural Comput. 10:5 (1998), 1299-1319.

[Schölkopf et al. 1999] B. Schölkopf, A. Smola, and K.-R. Müller, "Kernel principal component analysis", Chapter 20, pp. 327-352 in Advances in kernel methods, edited by C. J. C. Burges et al., MIT, 1999.

[Tenenbaum et al. 2000] J. B. Tenenbaum, V. de Silva, and J. C. Langford, "A global geometric framework for nonlinear dimensionality reduction", Science 290:5500 (2000), 2319-2323. 
[Wang 2014] Q. Wang, "Kernel principal component analysis and its applications in face recognition and active shape models", preprint, 2014. arXiv

[Zhu et al. 2018] J. Zhu, R. Balieu, X. Lu, and N. Kringos, "Microstructure evaluation of polymermodified bitumen by image analysis using two-dimensional fast Fourier transform", Mater. Design 137 (2018), 164-175.

[Zimmer et al. 2015] V. A. Zimmer, K. Lekadir, C. Hoogendoorn, A. F. Frangi, and G. Piella, "A framework for optimal kernel-based manifold embedding of medical image data", Comput. Med. Imag. Grap. 41 (2015), 93-107.

Received 6 Jan 2018. Revised 20 Mar 2018. Accepted 15 May 2018.

ELENA LOPEZ: elena.lopez-tomas@ec-nantes.fr

Institut de Calcul Intensif, École Centrale de Nantes, Nantes, France

ADRIEN SCHEUER: adrien.scheuer@ec-nantes.fr

Institut de Calcul Intensif, École Centrale de Nantes, Nantes, France

and

Institute of Information and Communication Technologies, Electronics and Applied Mathematics, Université catholique de Louvain, Louvain-la-Neuve, Belgium

EMMANUELLE ABISSET-CHAVANNE: emmanuelle.abisset-chavanne@ec-nantes.fr Institut de Calcul Intensif, École Centrale de Nantes, Nantes, France

FRANCISCO CHINESTA: francisco.chinesta@ensam.eu

Procédés et Ingénierie en Mécanique et Matériaux, Arts et Métiers ParisTech, Paris, France 
EDITORIAL BOARD

ANTONIO CARCATERRA

ERIC A. CARLEN

FRANCESCO DELL'ISOLA

RAFFAELE ESPOSITO

ALBERT FANNJIANG

Gilles A. FranCFORT

Pierangelo MARCATI

JEAN-JACQUES MARIGO

PETER A. MARKOWICH

MARTIN OSTOJA-STARZEWSKI

PIERRE SEPPECHER

DAVID J. STEIGMANN

PAUl STEINMANN

PierRe M. SuQueT

MANAGING EDITORS

MICOL AMAR

CORRADO LATTANZIO

ANGELA MADEO

MARTIN OSTOJA-STARZEWSKI

ADVISORY BOARD

ADNAN AKAY

Holm AltenBaCH

MICOL AMAR

HARM ASKES

TEODOR ATANACKOVIĆ

VICTOR BERDICHEVSKY

GUY BOUCHITTÉ

ANDREA BRAIDES

ROBERTO CAMASSA

MAURO CARFORE

ERIC DARVE

FELIX DARVE

ANNA DE MASI

GianPiEtro DEL Piero

EMMANUELE Di BENEDETTO

BERNOLD FIEDLER

IRENE M. GAMBA

DAVID Y. GAO

SERGEY GAVRILYUK

TIMOTHY J. HEALEY

DOMINIQUE JEULIN

ROGER E. KHAYAT

CORRADO LATTANZIO

ROBERT P. LIPTON

ANGELO LUONGO

ANGELA MADEO

JUAN J. MANFREDI

CARLO MARCHIORO

GÉRARD A. MAUGIN

ROBERTO NATALINI PATRIZIO NEFF

ANDREY PIATNITSKI

ERRICO PRESUTTI

MARIO PULVIRENTI

LUCIO RUSSO

Miguel A. F. SANJUAN

PATRICK SElVADURAI

ALEXANDER P. SEYRANIAN

MIROSLAV ŠILHAVÝ

GUIDO SWEERS

ANTOINETTE TORDESILLAS

LEV TRUSKINOVSKY

JUAN J. L. VELÁZQUEZ VINCENZO VESPRI ANGELO VULPIANI msp.org/memocs

Università di Roma "La Sapienza", Italia

Rutgers University, USA

(CO-CHAIR) Università di Roma "La Sapienza", Italia

(TREASURER) Università dell'Aquila, Italia

University of California at Davis, USA

(CO-CHAIR) Université Paris-Nord, France

Università dell'Aquila, Italy

École Polytechnique, France

DAMTP Cambridge, UK, and University of Vienna, Austria

(CHAIR MANAGING EDITOR) Univ. of Illinois at Urbana-Champaign, USA

Université du Sud Toulon-Var, France

University of California at Berkeley, USA

Universität Erlangen-Nürnberg, Germany

LMA CNRS Marseille, France

Università di Roma "La Sapienza", Italia

Università dell'Aquila, Italy

Université de Lyon-INSA (Institut National des Sciences Appliquées), France

(CHAIR MANAGING EDITOR) Univ. of Illinois at Urbana-Champaign, USA

Carnegie Mellon University, USA, and Bilkent University, Turkey

Otto-von-Guericke-Universität Magdeburg, Germany

Università di Roma "La Sapienza", Italia

University of Sheffield, UK

University of Novi Sad, Serbia

Wayne State University, USA

Université du Sud Toulon-Var, France

Università di Roma Tor Vergata, Italia

University of North Carolina at Chapel Hill, USA

Università di Pavia, Italia

Stanford University, USA

Institut Polytechnique de Grenoble, France

Università dell'Aquila, Italia

Università di Ferrara and International Research Center MEMOCS, Italia

Vanderbilt University, USA

Freie Universität Berlin, Germany

University of Texas at Austin, USA

Federation University and Australian National University, Australia

Université Aix-Marseille, France

Cornell University, USA

École des Mines, France

University of Western Ontario, Canada

Università dell' Aquila, Italy

Louisiana State University, USA

Università dell'Aquila, Italia

Université de Lyon-INSA (Institut National des Sciences Appliquées), France University of Pittsburgh, USA

Università di Roma "La Sapienza”, Italia

Université Paris VI, France

Istituto per le Applicazioni del Calcolo "M. Picone", Italy

Universität Duisburg-Essen, Germany

Narvik University College, Norway, Russia

Università di Roma Tor Vergata, Italy

Università di Roma "La Sapienza”, Italia

Università di Roma “Tor Vergata”, Italia

Universidad Rey Juan Carlos, Madrid, Spain

McGill University, Canada

Moscow State Lomonosov University, Russia

Academy of Sciences of the Czech Republic

Universität zu Köln, Germany

University of Melbourne, Australia

École Polytechnique, France

Bonn University, Germany

Università di Firenze, Italia

Università di Roma La Sapienza, Italia

MEMOCS (ISSN 2325-3444 electronic, 2326-7186 printed) is a journal of the International Research Center for the Mathematics and Mechanics of Complex Systems at the Università dell'Aquila, Italy.

Cover image: "Tangle” by $\odot$ John Horigan; produced using the Context Free program (contextfreeart.org).

PUBLISHED BY

7 mathematical sciences publishers

nonprofit scientific publishing

http://msp.org/

(C) 2018 Mathematical Sciences Publishers 
Mathematics and Mechanics of Complex Systems vol. 6 no. 3

The variational structure of classical plasticity

Gianpietro Del Piero

Far-reaching Hellenistic geographical knowledge hidden in

Ptolemy's data

\section{Lucio Russo}

Generation of SH-type waves due to shearing stress discontinuity in an anisotropic layer overlying an initially stressed elastic half-space

Santosh Kumar and Dinbandhu Mandal

Strain gradient and generalized continua obtained by homogenizing frame lattices

Houssam Abdoul-Anziz and Pierre Seppecher

On the effect of phase transition on the manifold dimensionality: application to the Ising model

Elena Lopez, Adrien Scheuer, Emmanuelle Abisset-Chavanne and Francisco Chinesta

MEMOCS is a journal of the International Research Center for the Mathematics and Mechanics of Complex Systems at the Università dell' Aquila, Italy.

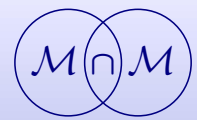

